Article

\title{
Effect of Solid Forms on Physicochemical Properties of Valnemulin
}

\author{
Jinbo Ouyang ${ }^{1, *}$, Jian Chen ${ }^{1}$, Limin Zhou ${ }^{1}$, Fangze Han ${ }^{1}$ and Xin Huang ${ }^{2}$ (D) \\ 1 State Key Laboratory for Nuclear Resources and Environment, East China University of Technology, \\ Nanchang 330013, China; jian_chan@ecit.cn (J.C.); lmzhou@ecit.cn (L.Z.); fzhan@ecit.cn (F.H.) \\ 2 School of Chemical Engineering \& Technology, Tianjin University, Tianjin 300072, China; x_huang@tju.edu.cn \\ * Correspondence: ouyangjinbo@ecut.edu.cn; Tel.: +86-0791-8389-6550
}

Received: 14 November 2019; Accepted: 9 December 2019; Published: 16 December 2019

check for updates

\begin{abstract}
To improve the physicochemical properties of valnemulin (VLM), different solid forms formed by VLM and organic acids, including tartaric acid (TAR), fumaric acid (FUM), and oxalic acid (OXA), were successfully prepared and characterized by using differential scanning calorimetry (DSC), scanning electron microscope (SEM), X-ray powder diffraction (XRPD), and Fourier-transform infrared spectroscopy (FT-IR). The excess enthalpy $H_{\mathrm{ex}}$ between VLM and other organic acids was calculated by COSMOthermX software and was used to evaluate the probability of forming multi-component solids between VLM and organic acids. By thermal analysis, it was confirmed that multi-component solid forms of VLM were thermodynamically more stable than VLM itself. Through dynamic vapor sorption (DVS) experiments, it was found that three multi-component solid forms of VLM had lower hygroscopicity than VLM itself. Furthermore, the intrinsic dissolution rate of VLM and its multi-component forms was determined in one kind of acidic aqueous medium by using UV-vis spectrometry. It was found that the three multi-component solid forms of VLM dissolved faster than VLM itself.
\end{abstract}

Keywords: valnemulin; solid forms; dissolution rate; hygroscopicity

\section{Introduction}

Many pharmaceuticals fail clinical trials, mostly due to pure physicochemical properties, including poor dissolution rate, low crystallinity, and strong hygroscopicity [1,2]. Thus, the discovery of methods that can improve the dissolution rate and stability of active pharmaceutical ingredient (API) is an important challenge in the pharmaceutical industry. Some of the methods currently being used include the discovery of new polymorphs, amorphous forms, solid dispersions, cocrystals, salt forms, and inclusion complexes [3-6]. However, the most popular method used to increase the dissolution rate and improve physicochemical properties is salt or cocrystal formation $[7,8]$. In fact, at least $50 \%$ of all active pharmaceutical ingredients (APIs) have been developed as multi-component solid forms, such as pharmaceutically acceptable salts $[9,10]$. Pharmaceutically acceptable salt counterparts can be classified into anions and cations. Anions include inorganic acids, sulfonic acids, carboxylic acids, anion amino acids, hydroxyl acids, fatty acids. Cations mainly refer to the cationic amino acids [11]. In recent years, pharmaceutical cocrystals also open a new door for solid forms that can enable substantial modification of physicochemical properties of API [12-14]. Pharmaceutical cocrystals are multi-component crystalline forms made of neutral molecular components, usually involving an API and one cocrystal former, also exhibiting a definite stoichiometry ratio, often leading to a hydrogen-bonded molecular complex $[15,16]$. The main distinction between salts and cocrystals is the charge transfer ratio occurring among the API and the guest molecule. If the proton involved 
in the hydrogen-bonding interaction is transferred from the donor to the acceptor, a salt is formed. On the contrary, a cocrystal is generated $[17,18]$.

Many methods and technologies have been developed for the preparation of pharmaceutical salts or cocrystals [19-21]. Solution-based reactive crystallization is one of the most efficient methods since crystallization is an important separation and purification process employed to produce a broad variety of materials in pharmaceutical, food, fine chemicals, and bulk chemical industries [22,23]. In the pharmaceutical industry, the crystallization process is usually designed to obtain pharmaceutical crystalline products with high purity [24].

Valnemulin (VLM, $\mathrm{C}_{31} \mathrm{H}_{52} \mathrm{~N}_{2} \mathrm{O}_{5} \mathrm{~S}$, molar mass: $564.78 \mathrm{~g} / \mathrm{mol}$, Figure 1) is an animal antibiotic used to treat mycoplasma disease [25-27]. Because of poor aqueous solubility, the amorphous VLM and hydrochloride salt of VLM, with improved solubility and pharmacokinetics, have been marketed [28]. However, the stability of the amorphous product is not as good as the crystalline one. The amorphous product generally has stronger hygroscopicity than the crystalline product, which makes it difficult to be stored. Furthermore, valnemulin hydrochloride has a bad smell, and its powder is prone to float in the air, which will make people allergic. Thus, to improve the physicochemical properties of the drug, it is important to develop multi-component solid forms of VLM and to compare their physicochemical properties with the physicochemical properties of VLM itself.

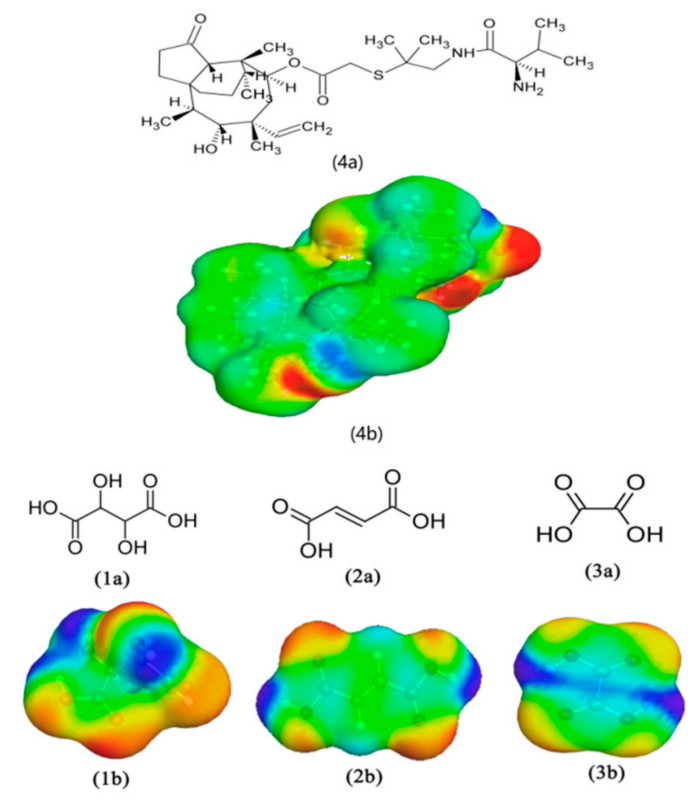

Figure 1. Molecular structure (1a-TAR, 2a-FUM, 3a-OXA, 4a-VLM) and 3D COSMO-surface screening charge densities (1b-TAR, 2b-FUM, 3b-OXA, 4b-VLM). VLM: valnemulin; TAR: tartaric acid; FUM: fumaric acid; OXA: oxalic acid.

Three multi-component solid forms of VLM with organic acids, including tartaric acid (TAR), fumaric acid (FUM), and oxalic acid (OXA), were successfully prepared. The excess enthalpy $H_{\mathrm{ex}}$ between VLM and organic acids was calculated by COSMOthermX software and was used to evaluate the probability of forming multi-component solids between VLM and organic acids. X-ray powder diffraction (XRPD), scanning electron microscope (SEM), differential scanning calorimetry (DSC), and Fourier-transform infrared spectroscopy (FT-IR) were applied to characterize the multi-component solid forms of VLM and organic acids. The hygroscopicity of the multi-component solids was evaluated by the dynamic vapor sorption (DVS). Finally, to evaluate the dissolution properties of these products, the intrinsic dissolution rate of these solid forms was measured in $\mathrm{pH}=6.8$ aqueous medium at $37^{\circ} \mathrm{C}$. 


\section{Experimental Section}

\subsection{Materials}

Valnemulin with mass purity higher than 0.95 was purchased from Hubei Longxiang Chemical Co., Ltd. (Wuxue, Hubei, China), and it was used without further purification. Tartaric acid (TAR), fumaric acid (FUM), and oxalic acid (OXA) with mass purity higher than 0.99 were purchased from the Aladdin Reagent Co., Ltd. (Shanghai, China). Acetonitrile and butyl acetate with mass fraction purity higher than 0.99 were purchased from Jiangxi Ganyi Chemical Co., Ltd. (Nanchang, Jiangxi, China).

\subsection{COSMO-RS Calculation}

The excess enthalpies $H_{\text {ex }}$ between VLM and organic acids were calculated by the COSMOtherm $X$ 3.0 program (COSMOlogic GmbH \& Co KG, Leverkusen, Germany). The COSMO input files of organic acids were taken from the COSMO database. The COSMO input file of VLM was obtained from quantum chemical calculations performed on the density functional theory (DFT) level using the DMOL3 module within Materials Studio (v 6.0, Accelrys, California, USA) package.

COSMO-RS is widely applied for predicting the formation of cocrystals/salts, and it contains two calculation steps $[29,30]$. Firstly, structures of API and co-formers should be optimized by quantum chemical calculations, and the COSMO model is applied to simulate a virtual conductor environment for all molecules [31-34]. Secondly, the molecular interaction energy should be determined based on electrostatics and hydrogen bonding $[35,36]$. So, COSMO-RS was used to simulate a virtually supercooled liquid mixture of API and co-former, and then the excess enthalpy $\left(H_{\mathrm{ex}}\right)$ of the mixture with a stoichiometric ratio of $m: n$ from pure components $A$ and $B$ was calculated:

$$
H_{e x}=H_{A B}-x_{m} H_{\text {pure }, A}-x_{n} H_{\text {pure }, B}
$$

where $H_{\text {pure }}$ and $H_{A B}$ refer to molar enthalpies in pure state and in $m: n$ mixture, and mole fraction can be calculated by $x_{m}=m /(m+n)$ and $x_{n}=n /(m+n)$. $H_{e x}$ refers to excess enthalpy, and compounds with $H_{\mathrm{ex}}<0$ means that there is a strong interaction between API and co-former, which will lead them to cocrystallize from solution. So, it is reasonable to apply excess enthalpy $H_{\mathrm{ex}}$ as a guide for predicting cocrystal/salts.

\subsection{Preparation of VLM Multi-Component Solids.}

VLM-TAR (1: 1). $2 \mathrm{~g}$ valnemulin and $0.5 \mathrm{~g}$ tartaric acid (1: 1 molar ratio) were dissolved into $50 \mathrm{~mL}$ acetonitrile at $60^{\circ} \mathrm{C}$. The slurry was reacted for $2 \mathrm{~h}$ under the stirring rate of $300 \mathrm{rpm}$. The temperature of the solution was cooled to $20^{\circ} \mathrm{C}$ at the rate of $10^{\circ} \mathrm{C} / \mathrm{h}$. The solid was crystallized out from the solution upon $40^{\circ} \mathrm{C}$, then was filtered and dried at $50^{\circ} \mathrm{C}$.

VLM-FUM (1: 1). $1 \mathrm{~g}$ valnemulin and $0.25 \mathrm{~g}$ fumaric acid (1:1 molar ratio) were dissolved into $25 \mathrm{~mL}$ butyl acetate at $60^{\circ} \mathrm{C}$. The slurry was reacted for $3 \mathrm{~h}$ under the stirring rate of $400 \mathrm{rpm}$. The temperature of the solution was cooled to $30^{\circ} \mathrm{C}$ at the rate of $10^{\circ} \mathrm{C} / \mathrm{h}$. The solid was crystallized out from the solution upon $45^{\circ} \mathrm{C}$, then was filtered and dried at $50^{\circ} \mathrm{C}$.

VLM-OXA ( $1: 1) .1 \mathrm{~g}$ valnemulin and $0.25 \mathrm{~g}$ oxalic acid $(1: 1 \mathrm{molar}$ ratio) were dissolved into $25 \mathrm{~mL}$ of butyl acetate at $60{ }^{\circ} \mathrm{C}$. The slurry was reacted for $3 \mathrm{~h}$ under the stirring rate of $400 \mathrm{rpm}$. The temperature of the solution was cooled to $20^{\circ} \mathrm{C}$ at the rate of $10^{\circ} \mathrm{C} / \mathrm{h}$. The solid was crystallized out from the solution upon $35^{\circ} \mathrm{C}$, then was filtered and dried at $50^{\circ} \mathrm{C}$.

\subsection{X-Ray Powder Diffraction}

To identify the solid form of VLM and its multi-component solids, XRPD of the obtained samples was measured by $\mathrm{Cu} \mathrm{K} \alpha$ radiation $\left(1.5405 \AA\right.$ ) between $2^{\circ}$ and $50^{\circ}$. The data collection was performed on Rigaku D/max-2500 (Rigaku, Tokyo, Japan). 


\subsection{Thermal Analysis}

The DSC of VLM and its multi-component solids was measured by DSC (DSC 1/500, Mettler-Toledo, Zurich, Switzerland) under nitrogen protection with a heating rate of $10 \mathrm{~K} / \mathrm{min}$, and the sample amount was about $5 \mathrm{mg}$.

\subsection{FT-IR Spectroscopy}

Infrared measurements were performed on a $\mathrm{KBr}$ disk. Fourier transformation infrared (FT-IR) spectra were recorded using a Bruker TENSOR 27 spectrometer (Karlsruhe, Germany) in the range of $400-4000 \mathrm{~cm}^{-1}$. All spectra were the results of averaging 16 scans, and the resolution was $2 \mathrm{~cm}^{-1}$.

\subsection{Dynamic Vapor Sorption (DVS)}

Dynamic vapor sorption experiments were performed on a DVS instrument (VTI-SA ${ }^{+}, \mathrm{TA}, \mathrm{New}$ Castle, USA). Samples were studied over a humidity range from 0 to $90 \% \mathrm{RH}$ at $25^{\circ} \mathrm{C}$, and the moisture sorption behavior of the samples was determined by continuously measuring the weight change of the samples at each humidity step. Each humidity step was made if less than $0.02 \%$ mass change occurred over $10 \mathrm{~min}$, with a maximum holding time of $3 \mathrm{~h}$.

\subsection{Intrinsic Dissolution Rate}

Intrinsic dissolution rate (IDR) was measured by a USP-certified Electrolab (RC-6, GUOMING, Tianjin, China). The measurement procedure was taken from a previous paper published by Shah [37], and it also met the requirement of the pharmacopeia. Solid with the amount of $200 \mathrm{mg}$ was put into the tablet press and compressed into a tablet. Then, the tablet was immersed into a $900 \mathrm{~mL}$ buffer medium with $\mathrm{pH}=6.8$ at $37^{\circ} \mathrm{C}$. The paddle speed was set at $75 \mathrm{rpm}$. At regular intervals of $10 \mathrm{~min}, 5 \mathrm{~mL}$ of the dissolution medium was withdrawn and replaced by an equal volume of fresh medium to maintain a constant volume. Before measurements, the absorption wavelength of VLM and multi-component solids was determined, and results revealed that there was no difference in absorption wavelength between VLM and multi-component solids. So, $210 \mathrm{~nm}$ was chosen as the absorption wavelength for VLM and multi-component solids. Samples were filtered through a $0.2 \mu \mathrm{m}$ nylon filter, and the concentration of samples was measured at $210 \mathrm{~nm}$ on the UV-vis spectrometer. The measured concentration $(\mathrm{mg} / \mathrm{L})$ was then changed into a cumulative amount dissolved $(\%)$. The cumulative amount dissolved was plotted as a function of time to produce the dissolution profile.

In order to have a better comparison, the amount of drug dissolved in the first $10 \mathrm{~min}\left(\mathrm{Q}_{10} / \%\right)$ and the time required for the dissolution of $50 \%$ drug $\left(\mathrm{T}_{50 \%} / \mathrm{min}\right)$ were selected. In addition, the similarity factor $f_{2}$ was applied to investigate the similarity between different dissolution profiles. It was defined by the following equation [38].

$$
f_{2}=50 \times \log \left\{\left[1+\frac{1}{n} \sum_{t=1}^{n}\left(R_{t}-T_{t}\right)^{2}\right]^{-0.5} \times 100\right\}
$$

where $n$ is the number of time points, $R_{\mathrm{t}}$ is the amount of dissolved reference at time $t$, and $T_{\mathrm{t}}$ is the amount of the dissolved tested sample at the same time. $f_{2}$ value between 50 and 100 indicates a similarity between two dissolution profiles, and the higher value means higher similarity [39].

\section{Results and Discussion}

\subsection{COSMO-RS Calculation}

Seven organic acids were selected to investigate the possibility of forming multi-component solids with VLM. But only three multi-component solids of VLM with acids, including tartaric acid, fumaric acid, and oxalic acid, were successfully prepared. The molecular structure and 3D COSMO-surface 
input files of these three acids are illustrated in Figure 1. Since the 3D COSMO-surface of VLM was not stored in the COSMO database, it was obtained from quantum chemical calculations, and the calculated result is also depicted in Figure 1. The excess enthalpy $H_{\mathrm{ex}}$ between VLM and acids was calculated by using the COSMO-surface of VLM and organic acids by the COSMOthermX 3.0 program, and the calculated results are depicted in Table 1. As mentioned before, the excess enthalpy $H_{\mathrm{ex}}$ between an API and co-former reflects the tendency of those two compounds to form salts or cocrystals. The high value of negative $H_{\mathrm{ex}}$ means that it's easy to form an API-co-former multi-component solid. According to data presented in Table 1, tartaric acid, oxalic acid, and fumaric acid were prone to form multi-component solid forms with VLM than the other four organic acids since the values of $H_{\text {ex }}$ between VLM and TAR, VLM and FUM, VLM and OXA were $-5.31 \mathrm{kcal} / \mathrm{mol},-4.90 \mathrm{kcal} / \mathrm{mol}$, and $-6.89 \mathrm{kcal} / \mathrm{mol}$, respectively. These results indicated that aliphatic dicarboxylic acids were more likely to interact with VLM molecule and form stable crystalline multi-component solids than aromatic carboxylic acids. The shorter carbon chain of the aliphatic dibasic acids might be responsible for the stronger interaction between VLM and acid molecules and thus resulted in the higher capability to form cocrystals/salts.

Table 1. The calculated excess enthalpy $H_{\mathrm{ex}}$ between VLM (valnemulin) and organic acids in 1 : 1 stoichiometry.

\begin{tabular}{cc}
\hline Co-Former & $\boldsymbol{H}_{\mathbf{e x}} \mathbf{( k c a l / m o l )}$ \\
\hline Oxalic acid & -6.89 \\
Tartaric acid & -5.31 \\
Fumaric acid & -4.90 \\
Succinic acid & -3.44 \\
Salicylic acid & -3.29 \\
Hexanedioic acid & -2.83 \\
Isonicotinic acid & -1.15 \\
\hline
\end{tabular}

\section{2. $X R P D / D S C / S E M$}

The multi-component solids of VLM were successfully prepared. The experiments were repeated three times, and its yields for VLM-TAR, VLM-FUM, VLM-OXA were $85 \%, 73 \%$, and $78 \%$, respectively. $\mathrm{X}$-ray powder diffraction patterns for organic acids and VLM and its multi-component solids are shown in Figure 2. The XRPD data showed that all of VLM multi-component solids displayed crystal characterization. The thermal analysis of organic acid and VLM and its multi-component solids was carried out by DSC, and the results are shown in Figure 3. The calculated thermodynamic parameters are presented in Table 2. As depicted in Figure 3, amorphous VLM had no certain melting point and exhibited a small endothermic step at $50{ }^{\circ} \mathrm{C}$, which corresponded to the glass transition temperature. Three crystalline multi-component solids exhibited an apparent melting point at $175^{\circ} \mathrm{C}$ for VLM-TAR, $130{ }^{\circ} \mathrm{C}$ for VLM-FUM, and $125^{\circ} \mathrm{C}$ for VLM-OXA, respectively. These data indicated that the multi-component solids had better thermal stability than VLM. To determine the similar polymorph of prepared samples, the XRPD and DSC were tested for samples obtained in the same method again, and the results are shown in Figures S1 and S2. Results indicated that no polymorph occurred, and all of them cocrystallized in the same polymorph. Figure 4 shows the crystal morphology of multi-component solids of VLM. Both of VLM-TAR and VLM-OXA were rod-like, while VLM-FUM was block-like. 


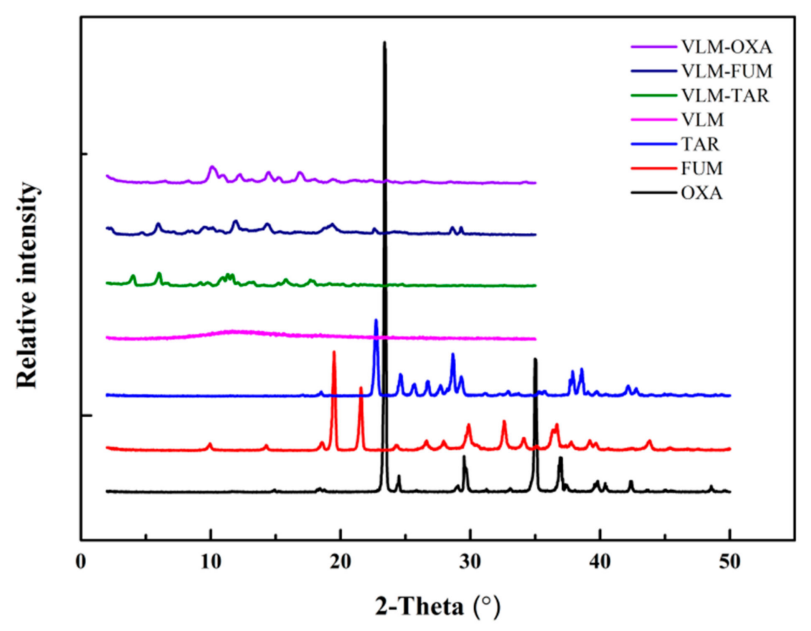

Figure 2. X-ray powder diffraction (XRPD) patterns of organic acids and VLM and its multicomponent systems.

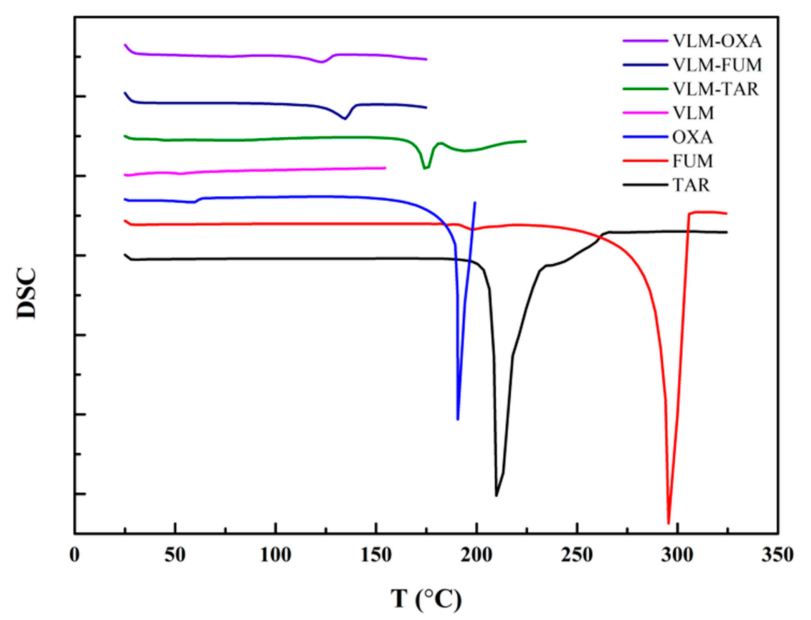

Figure 3. Differential scanning calorimetry (DSC) plots of organic acids and VLM and its multicomponent systems.

Table 2. The thermodynamic parameters of VLM and its multi-component solid forms.

\begin{tabular}{cccc}
\hline Samples & Onset $\left({ }^{\circ} \mathbf{C}\right)$ & Endset $\left({ }^{\circ} \mathbf{C}\right)$ & $\boldsymbol{T}_{\mathbf{m}}\left({ }^{\circ} \mathbf{C}\right)$ \\
\hline TAR & 200 & 230 & 210 \\
FUM & 250 & 310 & 295 \\
OXA & 170 & 200 & 188 \\
VLM & No peak & No peak & No peak \\
VLM-TAR & 170 & 180 & 175 \\
VLM-FUM & 120 & 135 & 130 \\
VLM-OXA & 120 & 135 & 125 \\
\hline
\end{tabular}

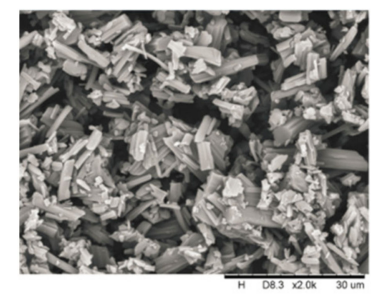

VLM-TAR

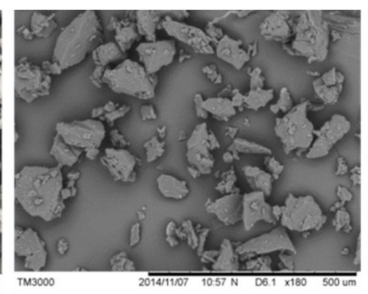

VLM-FUM

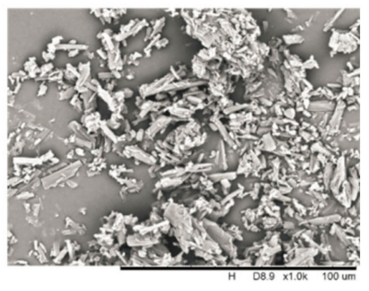

VLM-OXA

Figure 4. SEM images of VLM multi-component solids. 


\subsection{FT-IR Spectroscopy}

The FTIR spectra of VLM, co-formers, and multi-component solids are illustrated in Figures S3-S5. The detailed peaks assignment for the free VLM and each multi-component solid are given in Table 3. VLM showed a peak corresponding to -NH- stretching at $3350 \mathrm{~cm}^{-1}$ (Figure S3). The peak shifted to a higher wavenumber, $3355 \mathrm{~cm}^{-1}, 3435 \mathrm{~cm}^{-1}, 3400 \mathrm{~cm}^{-1}$ in the spectra of VLM-TAR, VLM-FUM, and VLM-OXA, respectively. The spectrum of TAR showed -COO- vibration peaks at 761 and $1750 \mathrm{~cm}^{-1}$, which also appeared in the spectra of VLM-TAR multi-component, as shown in the inset of Figure S3. The spectrum of FUM showed a -COO- vibration peak at $650 \mathrm{~cm}^{-1}$, which also appeared in the spectrum of VLM-FUM multi-component, as shown in the inset of Figure S4. The spectrum of OXA showed a -COO- vibration peak at $724 \mathrm{~cm}^{-1}$, which also appeared in the spectrum of VLM-OXA multi-component, as shown in the inset of Figure S5. These results indicated that blue shift really occurred in the -COO- vibration, which means strong stability of multi-component solids due to the hydrogen bonds between -NH- of VLM and -COOH- of co-formers.

Table 3. Relevant stretching bands in the FT-IR spectra of VLM and its multi-component solid forms ( $\left.\mathrm{cm}^{-1}\right)$.

\begin{tabular}{ccccc}
\hline Samples & $v_{\mathrm{N}-\mathrm{H}}$ & $\boldsymbol{v}_{\mathrm{C}=\mathrm{O}}$ & Co-Formers- $_{\mathbf{C O O H}}$ & ${\text { Multi-Component- } \boldsymbol{~ C O O}^{-}}^{-}$ \\
\hline VLM & 3350 & $1750 / 1650$ & & \\
TAR & & & $1750 / 761$ & \\
FUM & & & $1675 / 650$ & \\
OXA & & $1675 / 745$ & 761 \\
VLM-TAR & 3355 & $1745 / 1645$ & & 650 \\
VLM-FUM & 3435 & $1700 / 1650$ & & 745 \\
VLM-OXA & 3400 & $1700 / 1635$ & & \\
\hline
\end{tabular}

\subsection{Dynamic Vapor Sorption (DVS) Analysis}

The DVS isotherms for VLM and its multi-component solids were determined at $25^{\circ} \mathrm{C}$. The results are summarized in Table S1 and graphically depicted in Figure 5. For all solid samples, the moisture contents in the measurement room increased with a rising of relative humidity from 0 to $90 \%$. From the results, VLM was found to be the highest hygroscopic solid form compared with the other three multi-component solid forms. For RH $=90 \%$, the amount of moisture adsorbed by VLM was $15 \%$, which was higher than the other three solid forms. Obviously, the moisture content changed sharply vs. RH for VLM solid, while it changed slightly for the other three crystalline multi-component solids in the total range of RH. Furthermore, the morphology of solid samples after DVS experiments had also been checked, and the amorphous VLM would become a gel, while multi-component solids just kept original shape. The results confirmed again that the three novel multi-component solids had better physical stability than pure VLM.

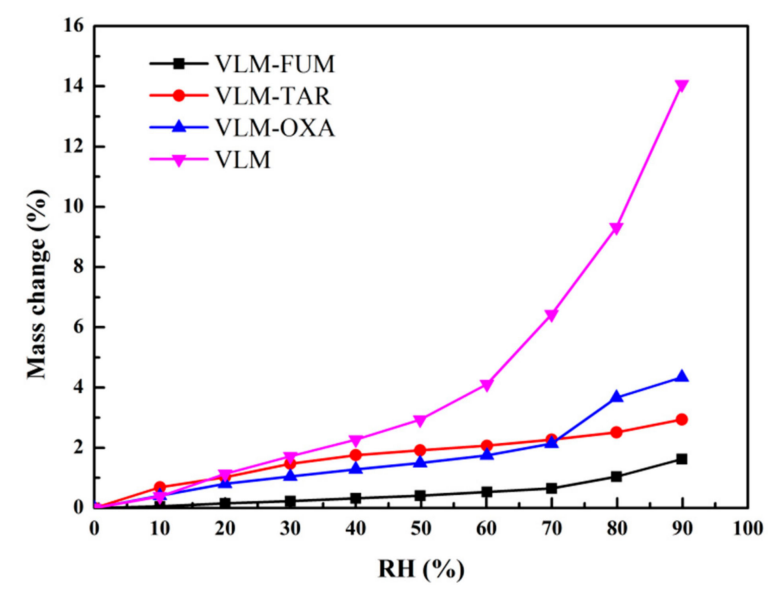

Figure 5. Dynamic vapor sorption isotherms of VLM and its multi-component systems at $25{ }^{\circ} \mathrm{C}$. 


\subsection{Intrinsic Dissolution Rate}

The intrinsic dissolution rate of VLM and its multi-component solid was measured by Electrolab under $\mathrm{pH}=6.8$ at $37^{\circ} \mathrm{C}$, and solution concentration was determined by the UV-vis spectrum. The UV-vis absorption coefficient ( $\xi$ ) was determined to be $0.0276,0.0165,0.0397$, and 0.0202 for VLM, VLM-TAR, VLM-FUM, and VLM-OXA, respectively. Results of solution concentration and cumulative amount dissolved are depicted in Table S2 and graphically shown in Figure 6. The amount of drug dissolved in the first $10 \mathrm{~min}\left(\mathrm{Q}_{10} / \%\right)$ and the time required for the dissolution of $50 \%$ drug $\left(\mathrm{T}_{50 \%} / \mathrm{min}\right)$ are depicted in Table 4. The dissolution profile of VLM indicated a slow dissolution rate, with only $4.56 \%$ of the drug being dissolved in the first $10 \mathrm{~min}$, and it might need infinite time to dissolve $50 \%$ of the added drug. The total amount of VLM dissolved in $100 \mathrm{~min}$ was just over $10 \%$ of the initial amount added. So, multi-component solids of VLM dissolved faster than VLM itself. The amounts of the drug dissolved in the first 10 min were $17.69 \%, 20.54 \%$, and $30.54 \%$ for VLM-TAR, VLM-FUM, and VLM-OXA, respectively. The time required for dissolving $50 \%$ of the added drugs was $60 \mathrm{~min}$, $40 \mathrm{~min}$, and $25 \mathrm{~min}$ for VLM-TAR, VLM-FUM, and VLM-OXA, respectively. In addition, the total amounts of drugs dissolved in $100 \mathrm{~min}$ were $56.81 \%, 65.45 \%$, and $92.63 \%$ for VLM-TAR, VLM-FUM, and VLM-OXA, respectively (Figure 6 and Table S2). From the similarity factors, which are shown in Table 5, the pure VLM was different from all the tested multi-component solid forms of VLM $\left(f_{2}\right.$ was in the range of $10 \%-21 \%$ ). With respect to the recorded dissolution profiles in the case of three multi-component solid forms of VLM, the similarity factor test revealed similar dissolution profiles for the VLM-TAR, VLM-FUM, and VLM-OXA ( $f_{2}$ was in the range from $50 \%$ to $80 \%$ ). However, it is important to note that the recorded $\mathrm{Q}_{10}$ value for VLM-OXA was higher than other multi-component solid forms of VLM. This could be explained by the fact that the chosen oxalic acid had a higher solubility than fumaric acid and tartaric acid. The above results demonstrated that the solubility and dissolution rate of APIs could be significantly increased by the formation of multi-component solid forms of API with other acids.

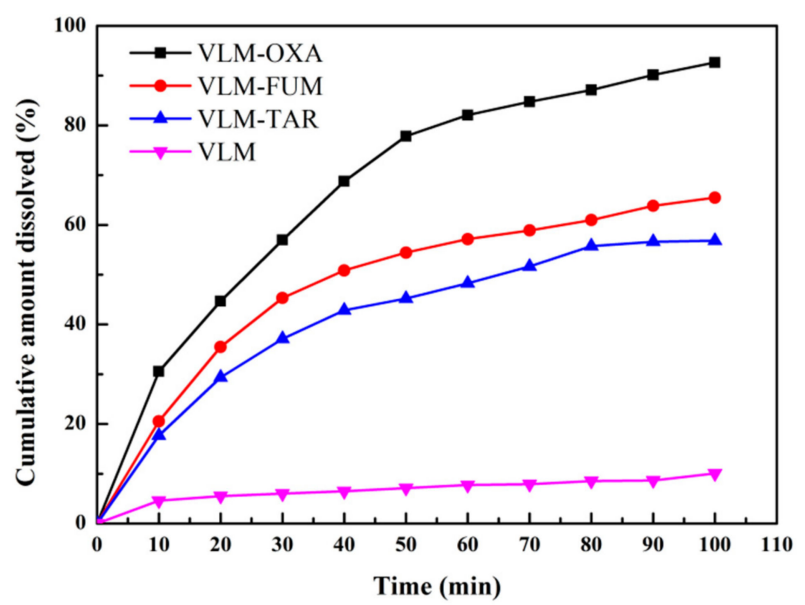

Figure 6. Intrinsic dissolution rate (IDR) of VLM and its multi-component solids in $\mathrm{pH}=6.8$ medium at $37^{\circ} \mathrm{C}$.

Table 4. The $\mathrm{Q}_{10 \min }$ and $\mathrm{T}_{50 \%}$ values of VLM and its multi-component solid forms.

\begin{tabular}{ccc}
\hline Samples & $\mathbf{Q}_{\mathbf{1 0 \operatorname { m i n }}} \mathbf{( \% )}$ & $\mathbf{T}_{\mathbf{5 0} \%} \mathbf{( m i n )}$ \\
\hline VLM & 4.56 & $\infty$ \\
VLM-TAR & 17.69 & 60 \\
VLM-FUM & 20.54 & 40 \\
VLM-OXA & 30.54 & 25 \\
\hline
\end{tabular}


Table 5. Comparison of the dissolution curves of VLM and its multi-component solid forms by using the $f_{2}$ similarity factor $\left(f_{2}\right.$ between 50 and 100 indicates a similarity between two dissolution profiles).

\begin{tabular}{cc}
\hline Samples Compared & $f_{2}$ \\
\hline VLM-TAR:VLM & 21.80 \\
VLM-FUM:VLM & 18.07 \\
VLM-OXA:VLM & 9.76 \\
VLM-FUM:VLM-TAR & 78.11 \\
VLM-OXA:VLM-FUM & 57.17 \\
VLM-OXA:VLM-TAR & 60.74 \\
\hline
\end{tabular}

\section{Conclusions}

In this work, three multi-component solid forms of VLM with organic acids were successfully prepared by solution crystallization method. The excess enthalpy $H_{\mathrm{ex}}$ between VLM and organic acids was calculated by the COSMO-RS theory, and results revealed that cocrystals/salts were more easily formed by VLM and three organic acids (TAR, FUM, and OXA). Compared with the amorphous VLM, the three multi-component products, including VLM-TAR, VLM-FUM, and VLM-OXA, were more stable due to crystalline state. The three multi-component solid forms also had a lower hygroscopicity than VLM, which was proved by the DVS analysis. So, it was more convenient to store the multi-component solids in a relatively flexible environment. Moreover, the multi-component solids displayed a faster dissolution rate and higher solubility than VLM. The above results indicated that the physicochemical properties of API could be improved by the multi-component formation of API with organic acids.

Supplementary Materials: The following are available online at http://www.mdpi.com/2073-4352/9/12/675/s1, Figure S1: XRPD patterns of VLM and multi-component solids, Figure S2: DSC curves of VLM and multi-component solids, Figure S3: FT-IR spectra of VLM and VLM-TAR multi-component form (inset shows the local amplification of a certain region), Figure S4. FT-IR spectra of VLM and VLM-FUM multi-component form (inset shows the local amplification of a certain region), Figure S5. FT-IR spectra of VLM and VLM-OXA multi-component form (inset shows the local amplification of a certain region), Table S1. Dynamic vapor sorption data for VLM and its multi-component solid forms at $25{ }^{\circ} \mathrm{C}$, Table S2. The intrinsic dissolution rate of VLM and its multi-component solid forms in $\mathrm{pH}=6.8$ aqueous medium at $37^{\circ} \mathrm{C}$.

Author Contributions: For research articles with several authors, a short paragraph specifying their individual contributions must be provided. The following statements should be used "conceptualization, J.O. and L.Z.; methodology, J.C.; software, X.H.; validation, F.H.; formal analysis, J.O.; investigation, J.O.; resources, J.O.; data curation, X.H.; writing — original draft preparation, J.O.; writing-review and editing, L.Z.; visualization, J.O.; supervision, J.O.; project administration, J.O.; funding acquisition, J.O.", please turn to the CRediT taxonomy for the term explanation. Authorship must be limited to those who have contributed substantially to the work reported.

Funding: The authors are grateful to the financial support of the National Natural Science Foundation of China (No. 21706028) and the Natural Science Foundation of Jiangxi Province (No. 20171BAB213016).

Conflicts of Interest: The authors declare no conflict of interest.

\section{References}

1. Leeson, P. Drug discovery: Chemical beauty contest. Nature 2012, 481, 455-456. [CrossRef] [PubMed]

2. Ouyang, J.B.; Wang, J.K.; Wang, Y.L.; Yin, Q.X.; Hong, H.X. Thermodynamic study on dynamic water and organic vapor sorption on amorphous valnemulin hydrochloride, Front. Chem. Sci. Eng. 2015, 9, 94-104.

3. Schultheiss, N.; Newman, A. Pharmaceutical cocrystals and their physicochemical properties. Cryst. Growth Des. 2009, 9, 2950-2967. [CrossRef] [PubMed]

4. Masuda, T.; Yoshihashi, Y.; Yonemochi, E.; Fujii, K.; Uekusa, H.; Terada, K. Cocrystallization and amorphization induced by drug-excipient interaction improves the physical properties of acyclovir. Int. J. Pharm. 2012, 422, 160-169. [CrossRef] [PubMed]

5. Morissette, S.L.; Almarsson, Ö.; Peterson, M.L.; Remenar, J.F.; Read, M.J.; Lemmo, A.V.; Ellis, S.; Cima, M.J.; Gardner, C.R. High-throughput crystallization: Polymorphs, salts, co-crystals and solvates of pharmaceutical solids. Adv. Drug Deliver Rev. 2004, 56, 275-300. [CrossRef] [PubMed] 
6. Carina, D.; Sergey, V.D.; Michael, S.; István, F. Polymer Swelling. Drug Mobilization and Drug Recrystallization in Hydrating Solid Dispersion Tablets Studied by Multinuclear NMR Microimaging and Spectroscopy. Mol. Pharm. 2011, 8, 1247-1256.

7. Geetha, B.; Ashiwini, N. Clofazimine Mesylate: A High Solubility Stable Salt. Cryst. Growth Des. 2012, 12, 6250-6259.

8. Flavia, A.M.; Mihaela, M.P.; Gheorghe, B.; Xenia, F.; Irina, K. Ketoconazole Salt and Co-crystals with Enhanced Aqueous Solubility. Cryst. Growth Des. 2013, 13, 4295-4304.

9. Gardner, D.R.; Almarsson, O.; Chen, H.; Morrisette, S.; Peterson, M.; Zhang, Z.; Wang, S.; Lemmo, A.; Gonzales-Zugasti, J.; Monagle, J.; et al. Application of high throughput technologies to drug substance and drug product development. J. Comput. Chem. Eng. 2004, 28, 943-953. [CrossRef]

10. Ballach, S.; Korn, C. Pharmaceutical evaluation of early development candidates "the $100 \mathrm{mg}$-approach". Int. J. Pharm. 2004, 275, 1-12. [CrossRef]

11. Jangmi, L.; Suzie, P.; Seon, J.Y.; Yong, W.J.; Youngjoo, B.; Soon, H.Y.; Min, K.J.; Sung, K.K.; Eun, H.L. Multicomponent System of NPS-1034, an Orally Administered Lung Cancer Drug Candidate, with Sulfonic Acids and Solid State Characterization. Cryst. Growth Des. 2013, 13, 3958-3968.

12. Ouyang, J.B.; Na, B.; Zhou, L.M.; Xiao, S.J.; Xiong, G.X.; Jin, T.X. Crystal structures and phase transformation of two novel solvates of valnemulin hydrochloride. CrystEngComm 2018, 20, 563-569. [CrossRef]

13. Guo, M.S.; Wang, K.; Qiao, N.; Yardley, V.; Li, M.Z. Investigating Permeation Behavior of Flufenamic Acid Cocrystals Using a Dissolution and Permeation System. Mol. Pharm. 2018, 15, 4257-4272. [CrossRef] [PubMed]

14. Ouyang, J.B.; Na, B.; Liu, Z.R.; Zhou, L.M.; Hao, H.X. Determination of Solubility and Nucleation Kinetics of Valnemulin Hydrochloride Solvate. J. Solut. Chem. 2019, 48, 413-426. [CrossRef]

15. Yun, H.; Katarzyna, G.; Andrea, E.; Patrick, M. Mechanochemical Reaction of Sulfathiazole with Carboxylic Acids: Formation of a Cocrystal, a Salt, and Coamorphous Solids. Cryst. Growth Des. 2014, 14, 803-813.

16. Hathwar, V.R.; Pal, R.; Guru Row, T.N. Charge Density Analysis of Crystals of Nicotinamide with Salicylic Acid and Oxalic Acid: An Insight into the Salt to Cocrystal Continuum. Cryst. Growth Des. 2010, 10, 3306-3310.

17. Wouters, J.; Quere, L.; Thurston, D.E. Pharmaceutical Salts and Cocrystals; Royal Society of Chemistry: Cambridge, UK, 2011.

18. Cecília, C.P.S.; Rebeka, D.O.; Juan, C.T.; Sara, B.H.; Alejandro, P.A.; Javier, E. The Continuum in 5-Fluorocytosine. Toward Salt Formation. Cryst. Growth Des. 2013, 13, 4315-43225.

19. Sharmarke, M.; Derek, A.T.; Martin, V.; Panagiotis, G.K.; Sarah, L.P. Salt or Cocrystal? A New Series of Crystal Structures Formed from Simple Pyridines and Carboxylic Acids. Cryst. Growth Des. 2009, 9, 2881-2889.

20. Pramod, K.G.; Ram, T.; Arunachalam, R. Multiple Crystal Forms of p-Aminosalicylic Acid: Salts, Salt Co-Crystal Hydrate, Co-Crystals, and Co-Crystal Polymorphs. Cryst. Growth Des. 2013, 13, 360-366.

21. Schultheiss, N.; Smit, J.P.; Hanko, J.A. Three isostructural solvates of finasteride and their solid-state characterization. Eur J Pharm Sci. 2009, 38, 498-503. [CrossRef]

22. Myerson, A. Handbook of Industrial Crystallization, 2nd ed.; Butterworth-Heinemann: Boston, USA, 2002.

23. Alvarez, A.J.; Myerson, A.S. Continuous Plug Flow Crystallization of Pharmaceutical Compounds. Cryst. Growth Des. 2010, 10, 2219-2228. [CrossRef]

24. Chen, J.; Sarma, B.; Evans, J.M.; Myerson, A.S. Pharmaceutical Crystallization. Cryst. Growth Des. 2011, 11, 887-895. [CrossRef]

25. Ouyang, J.B.; Wang, J.K.; Huang, X.; Gao, Y.; Bao, Y.; Wang, Y.; Yin, Q.; Hao, H. Gel Formation and Phase Transformation during the Crystallization of Valnemulin Hydrogen Tartrate. Ind. Eng. Chem. Res. 2014, 53, 16859-16863. [CrossRef]

26. Wattanaphansak, S.; Singer, R.S.; Gebhart, C.J. In vitro antimicrobial activity against 10 North American and European Lawsonia intracellularis isolates. Vet. Microbiol. 2009, 134, 305-310. [CrossRef] [PubMed]

27. Karlsson, M.; Oxberry, S.L.; Hampson, D.J. Antimicrobial susceptibility testing of Australian isolates of Brachyspira hyodysenteriae using a new broth dilution method. Vet. Microbiol. 2002, 84, 123-133. [CrossRef]

28. Qiu, S.H.; Jian, C.L.; Li, J.X.; Xi, X.; Peng, D.; Jian, Z.S.; Shuang, Y.D. Residue depletion of valnemulin in swine tissues after oral administration. Anal. Chim. Acta. 2010, 664, 62-67.

29. Klamt, A.; Eckert, F. COSMO-RS: A novel and efficient method for the a priori prediction of thermophysical data of liquids. Fluid Phase Equilib. 2000, 172, 43-72. [CrossRef] 
30. Klamt, A. The COSMO and COSMO-RS solvation models. Wiley Interdiscipl. Rev. Comput. Mol. Sci. 2011, 1, 699-709. [CrossRef]

31. Abramov, Y.A.; Christoph, L.; Andreas, K. Pharmaceutics, Preformulation and Drug Delivery: Rational Coformer or Solvent Selection for Pharmaceutical Cocrystallization or Desolvation. J. Pharm. Sci. 2012, 101, 3687-3697. [CrossRef]

32. Klamt, A. COSMO-RS: From Quantum Chemistry to Fluid Phase Thermodynamics and Drug Design; Elsevier: Amsterdam, The Netherlands, 2005.

33. Mohamed, S.; Tocher, D.A.; Price, S.L. Computational prediction of salt and co-crystal structuresDoes a proton position matter. Int. J. Pharm. 2011, 418, 187-198. [CrossRef]

34. Musumeci, D.; Hunter, C.A.; Prohens, R.; Scuderi, S.; McCabe, J.F. Virtual co-crystal screening. Chem Sci. 2011, 2, 883-890. [CrossRef]

35. Zhou, T.; Chen, L.; Ye, Y.M.; Chen, L.F.; Qi, Z.W.; Freund, H.; Sundmacher, K. An Overview of Mutual Solubility of Ionic Liquids and Water Predicted by COSMO-RS. Ind. Eng. Chem. Res. 2012, 51, 6256-6264. [CrossRef]

36. Eckert, F.; Klamt, A. Fast solvent screening via quantum chemistry: COSMO-RS approach. AIChE J. 2002, 48, 369-385. [CrossRef]

37. Shah, J.C.; Chen, J.R.; Chow, D. Metastable Polymorph of Etoposide with Higher Dissolution Rate. Drug Dev. Ind. Pharm. 1999, 25, 63-67. [CrossRef] [PubMed]

38. Costa, P. An alternative method to the evaluation of similarity factor indissolution testing. Int. J. Pharm. 2001, 220, 77-83. [CrossRef]

39. Lauretta, M.; Giovanna, B.; Mariarosa, M.; Andrea, C.; Andrea, C.; Ubaldo, C. II. Technological approaches to improve the dissolution behavior of nateglinide, a lipophilic insoluble drug: Co-milling. Int. J. Pharm. 2013, $454,568-572$.

(C) 2019 by the authors. Licensee MDPI, Basel, Switzerland. This article is an open access article distributed under the terms and conditions of the Creative Commons Attribution (CC BY) license (http://creativecommons.org/licenses/by/4.0/). 